\title{
DISEÑO DE UN PORTAL WEB QUE PERMITA ACCEDER A LOS RECURSOS Y SERVICIOS DEL OBSERVATORIO DE ECONOMÍA SOLIDARIA
}

EDNA MARGARITA REGINO ESTEBAN, OSMAN DARÍO OBREGÓN SILGADO, HENRY HERNÁNDEZ PADILLA, MARIO MEZQUIDA MENDOZA, SOHELY RUA CASTAÑEDA

Universidad Cooperativa de Colombia, sedes Montería y Bucaramanga
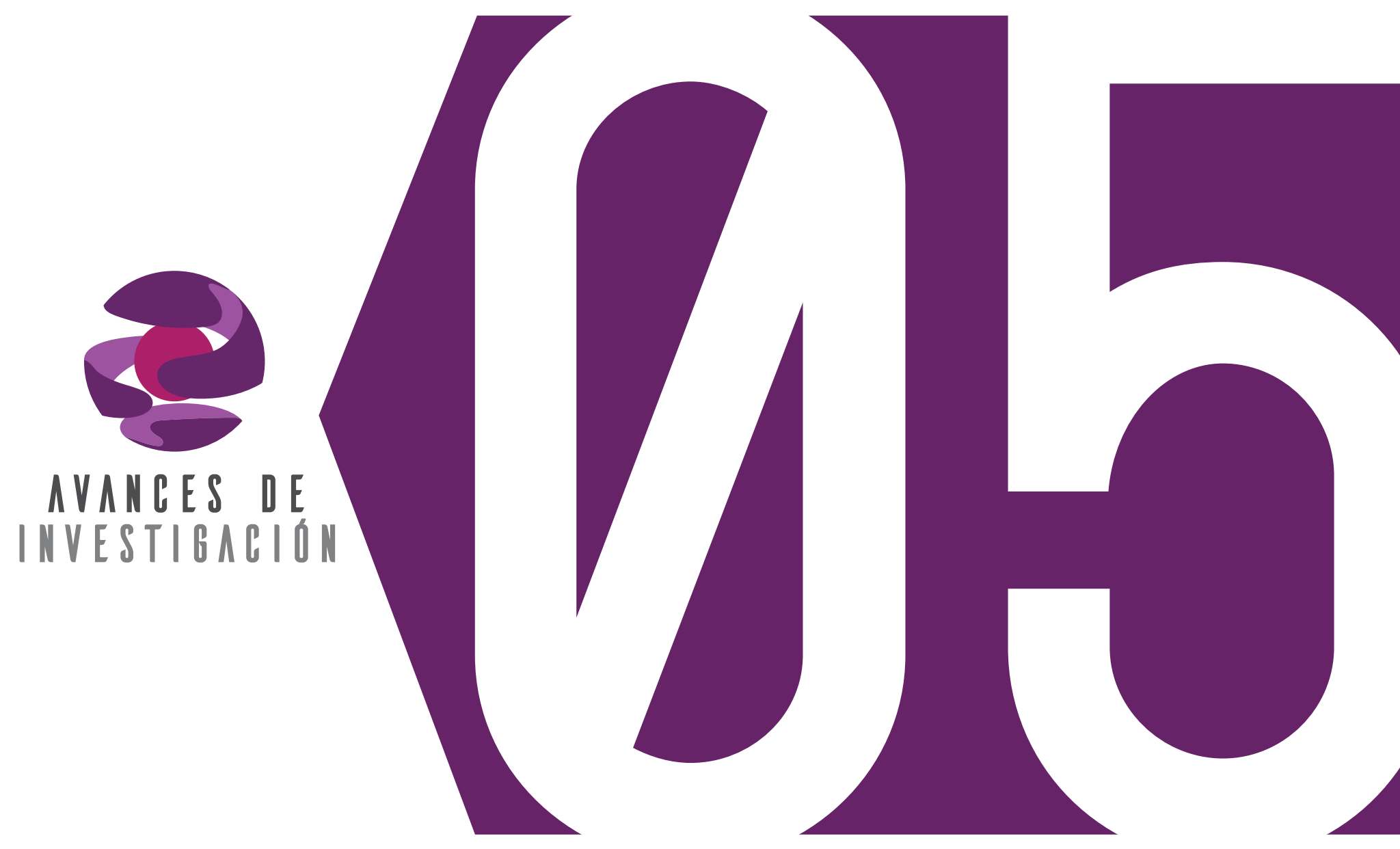

NOTA LEGAL

El presente documento de trabajo ha sido incluido dentro de nuestro repositorio institucional como Apropiación social de conocimiento por solicitud del autor, con fines informativos, educativos o académicos. Asimismo, los argumentos, datos y análisis incluidos en el texto son responsabilidad absoluta del autor y no representan la opinión del Fondo Editorial o de la Universidad.

\section{DISCLAIMER}

This working paper has been uploaded to our institutional repository as Social Appropriation of Knowledge due to the request of the author. This document should be used for informational, educational or academic purposes only. Arguments, data and analysis included in this document represent authors' opinion not the Press or the University. 


\section{ACERCA DE LOS AUTORES}

Edna Margarita Esteban Regino, magíster en Tecnología Educativa y Medios Innovadores para la Educación; profesora auxiliar del programa de Ingeniería de Sistemas, Universidad Cooperativa de Colombia, sede Montería.

Correo electrónico: edna.estebanr@campusucc.edu.co

Cvlac: https://scienti.minciencias.gov.co/cvlac/visualizador/generarCurriculoCv.do?cod_rh=0001637978

orcid: https://orcid.org/0000-0001-7790-4532

Google Scholar: https://scholar.google.com.tw/citations?hl=es\&user=qAosTJOAAAAJ

Osman Darío Obregón Silgado, estudiante del programa de Ingeniería de Sistemas, Universidad Cooperativa de Colombia, sede Montería.

Correo electrónico: osman.obregons@campusucc.edu.co

Cvlac: https://scienti.minciencias.gov.co/cvlac/visualizador/generarCurriculoCv.do?cod_rh=0001938879

orcid: https://orcid.org/0000-0003-3547-3679

Henry Hernández Padilla, estudiante del programa de Ingeniería de Sistemas, Universidad Cooperativa de Colombia, sede Montería.

Correo electrónico: henry.hernandezp@campusucc.edu.co

Cvlac: https://scienti.minciencias.gov.co/cvlac/visualizador/generarCurriculoCv.do?cod_rh=0001860355

orcid: https://orcid.org/0000-0002-3570-9488

Mario Miguel Mezquida Mendoza, estudiante del programa de Ingeniería de Sistemas, Universidad Cooperativa de Colombia, sede Montería.

Correo electrónico: mario.mezquidam@campusucc.edu.co

Cvlac: https://scienti.minciencias.gov.co/cvlac/visualizador/generarCurriculoCv.do?cod_rh=0001860140

orcid: https://orcid.org/0000-0002-0772-703X

Sohely Rua Castañeda, magíster en Educación y Desarrollo Humano, Universidad de Manizales; profesora, investigadora y coordinadora de la maestría en Economía Solidaria para el Desarrollo Territorial, Universidad Cooperativa de Colombia, sede Bucaramanga.

Correo electrónico: sohely.rua@campusucc.edu.co

Cvlac: https://scienti.minciencias.gov.co/cvlac/visualizador/generarCurriculoCv.do?cod_rh=0001221213 orcid: https://orcid.org/0000-0001-9591-9447

Este documento puede ser consultado, descargado o reproducido desde nuestro repositorio institucional (http://repository.ucc. edu.co/handle/20.500.12494/7369) para uso de sus contenidos, bajo la licencia de Creative Commons Reconocimiento-NoComercial-SinO braDerivada 4.0 Internacional. http://creativecommons.org/licenses/by-nc-nd/4.0/ 


\section{PERFIL DEL GRUPO DE INVESTIGACIÓN}

Este documento de trabajo ha sido realizado por investigadores de los grupos INDEsCo (Universidad Cooperativa de Colombia, sede Bogotá), seclam (Universidad Cooperativa de Colombia, sede Bucaramanga), en el área de las ciencias administrativas. La línea de investigación de la que surge el documento es economía solidaria y el proyecto asociado se titula: observatorio de impacto social, económico y ambiental de la economía solidaria en Colombia, código INv3008, vigencia 2021 y ha sido financiado por la Universidad Cooperativa de Colombia en la convocatoria de proyectos de mediana cuantía 2020-2

Gruplac Indesco:

https://sba.minciencias.gov.co/Buscador_HojasDeVida/BuscadorIFindIt/ busqueda? $q=$ indesco\&pagenum $=1 \&$ start=0\&type=load\&lang=es\&idss=5AnyAAHyyMHCpUh Gruplac SECLAM:

https://sba.minciencias.gov.co/Buscador_HojasDeVida/BuscadorIFindIt/ busqueda?q=seclam\&pagenum=1\&start=0\&type=load\&lang=es\&idss=tXTovRlI7C8x8dG

\section{CÓMO CITAR ESTE DOCUMENTO}

Esteban Regino, E. M., Obregón Silgado, O. D., Hernández Padilla, H., Mezquida Mendoza, M. M. y $\mathrm{Ru}$ Castañeda, S. (2021). Diseño de un portal web que permita acceder a los recursos y servicios del Observatorio de Economía Solidaria (Working papers N. ${ }^{\circ}$ 05). Ediciones Universidad Cooperativa de Colombia. doi: https://doi.org/10.16925/wpai.08 


\section{RESUMEN}

El presente documento integra los apartados concernientes a la investigación que tuvo como objetivo principal "Establecer los aspectos tecnológicos necesarios para el diseño de un portal web que permita acceder a los recursos y servicios del observatorio de economía solidaria, garantizando la conservación y actualización permanente de la información". Se describe la metodología propuesta para el logro de los objetivos, la cual consistió, principalmente, en realizar una revisión bibliográfica de artículos científicos en diversas bases de datos de acceso abierto (Scopus, Mendeley y Google académico) en las que se declaró un tiempo de 10 años de antigüedad. Se cuenta con el desarrollo del estado del arte y el marco conceptual integrado por conceptos como: observatorio, economía solidaria y portal web. Se realiza la presentación de resultados y se llega a la conclusión de que es necesario implementar todos aquellos requerimientos funcionales que ayuden al cumplimiento de sus funciones u obligaciones del observatorio. Para los requerimientos no funcionales, es relevante contar con una plataforma de colaboración electrónica basada en la web y móvil compatible con diferentes navegadores, con un sistema de información que dé soporte al almacenamiento de la información, la difusión de resultados, el procesamiento eficiente y apoyo a la toma de decisiones. Por otra parte, es importante no desconocer los desafíos a los que se enfrenta el desarrollo de un portal web: rendimiento de los algoritmos, aprendizaje automático, amigabilidad de la interfaz de usuario, recolección de los datos y actualización automática de la información.

Palabras clave: diseño, economía solidaria, observatorio digital, portal web. 


\section{TABLA DE CONTENIDO}

Introducción • 6

\section{Metodología $\cdot 7$}

Marco conceptual $\cdot 7$

8/ Observatorio

Resultados $\cdot 10$

10/ Aspectos tecnológicos
13 / Caracterización de observatorios de economía solidaria

14/ Prototipo no operacional o modelo no funcional del portal web del observatorio de economía solidaria

Conclusiones $\cdot 16$

Referencias $\cdot 17$ 


\title{
DISEÑO DE UN PORTAL WEB QUE PERMITA ACCEDER A LOS RECURSOS Y SERVICIOS DEL OBSERVATORIO DE ECONOMÍA SOLIDARIA
}

\author{
Edna Margarita Regino Esteban, Osman Darío \\ Obregón Silgado, Henry Hernández Padilla, Mario \\ Mezquida Mendoza, Sohely Rua Castañeda
}

\section{INTRODUCCIÓN}

El sector de la economía solidaria se reconoce por su capacidad de autogestión, derivada del trabajo y los aportes de sus asociados; de esta manera, se potencian sus habilidades individuales y se logran aportes sustanciales en la calidad de vida de las personas y sus comunidades. Es así como se han realizado diferentes esfuerzos por medir el impacto socioeconómico de las organizaciones del sector, entre ellos se encuentra el reporte de estados financieros que deben realizar las organizaciones a la Supersolidaria (Superintendencia de Economía Solidaria).

Ante esa necesidad, los gremios como Confecoop y Ascoop, la academia y los organismos internacionales han impulsado proyectos orientados a valorar tales impactos, mediante métodos cualitativos, cuantitativos o mixtos; también las organizaciones cada vez han incorporado modelos de rendición de cuentas buscando hacer visible el aporte que hacen a la sociedad; no obstante, el balance de este ejercicio indica que no han sido suficientes los estudios realizados, entre otras razones, porque cada uno define variables diferentes, se realizan en momentos del tiempo asimétricos y se usan instrumentos diversos, con lo cual se dificulta tener información comparable e impide aportar información a la toma de decisiones en las políticas públicas.

Ante dichas debilidades en la información y medición del impacto, se propuso en la Universidad Cooperativa de Colombia desarrollar el proyecto de investigación "Observatorio de impacto social, económico y ambiental de la economía solidaria en Colombia". Un observatorio se entiende como un centro de pensamiento especializado sobre un tema, que produce análisis periódicos que permiten comprender la evolución en el tiempo de dicho tema. En particular, los observatorios sociales están orientados a crear conocimiento derivado de una problemática social específica, siendo común encontrar observatorios en temas como derechos laborales, derechos humanos, violencia de género. Un observatorio contribuye a generar conocimiento en la medida en que puede hacer estudios longitudinales y puede agregar información con criterios sectoriales 
y regionales, dada la homogeneidad de los instrumentos de recolección de información de los que dispone.

Un aspecto clave de un observatorio es la publicación de resultados, que brinde información confiable a la comunidad, en general, academia, gremios, gobiernos, entre otros, razón por la cual, para alcanzar este propósito se definió un objetivo específico dentro del proyecto orientado a establecer los aspectos tecnológicos necesarios para el diseño de un portal web que permita acceder a los recursos y los servicios del observatorio de economía solidaria, garantizando la conservación y la actualización permanente de la información.

\section{METODOLOGÍA}

Para adelantar este objetivo específico sobre las características del portal web para el observatorio, se diseñó un microestudio de tipo documental, descriptivo y exploratorio (Hernández et al., 2006), que permitiera caracterizar observatorios existentes para reconocer metodologías que conlleven al diseño de una plataforma tecnológica de captura y procesamiento de información con reportes públicos; de manera específica, se identificaron observatorios de economía solidaria existentes en Colombia y en el mundo con el fin de recolectar buenas prácticas y experiencias significativas útiles en la adaptación metodológica que se requiere para el observatorio.

Se realizó una revisión bibliográfica de artículos científicos relacionados con palabras clave, como: observatorio virtual, observatorio digital, observación web, portales de conocimiento, observatorio social, observatorio tecnológico y observatorio, en diversas bases de datos de acceso abierto (Scopus, Mendeley y Google académico) en las que se declaró un intervalo de 10 años de antigüedad (Hernández et al., 2006; Esteban-Regino, 2016).

A partir de la revisión bibliográfica se identificaron variables de análisis que permiten la caracterización de cinco observatorios de economía solidaria existentes en Colombia y en el mundo, haciendo uso de la técnica del Benchmarking como una herramienta que permitiera tomar como referencia los mejores aspectos de otros observatorios (Briones-Veliz et al., 2021), para así obtener un prototipo no operacional o modelo no funcional del observatorio de economía solidaria, con el cual se pudieran probar ciertos aspectos del diseño (Kendall y Kendall, 2011). Dichos aspectos se validaron de acuerdo con las características de usabilidad aplicando un cuestionario en línea de seis preguntas de validación de usabilidad, como dice Pressman (2010), y que se puede observar en el anexo de la figura 9.

\section{MARCO CONCEPTUAL}

El marco conceptual proporcionará una idea clara de las palabras clave que permitieron alcanzar el objetivo propuesto: "Establecer los aspectos tecnológicos necesarios para el diseño de un portal web que permita
AVANCES DE INVESTIGACIÓN

DISEÑO DE UN PORTAL WEB QUE PERMITA ACCEDER A LOS RECURSOS Y SERVICIOS DEL OBSERVATORIO DE ECONOMIIA SOLIDARIA 
AVANCES DE INVESTIGACIÓN

DISEÑO DE UN PORTAL

WEB QUE PERMITA

ACCEDER A LOS

RECURSOS Y SERVICIOS

DEL OBSERVATORIO DE

ECONOMÍA SOLIDARIA acceder a los recursos y servicios del observatorio de economía solidaria, garantizando la conservación y actualización permanente de la información". Para ello se realizó una revisión documental, partiendo de la definición de economía solidaria del contexto para el cual se diseñará el observatorio, e identificando qué aspectos tecnológicos son necesarios para el diseño de un portal web.

\section{OBSERVATORIO}

Según Soler (2015), el término observatorio se ha relacionado con la observación de fenómenos astronómicos o astrológicos. En décadas recientes, se ha empezado a utilizar para analizar fenómenos sociales, por lo que la observación es un proceso fundamental en todo proceso de investigación y es entendido como una herramienta de monitoreo con técnicas cualitativas o cuantitativas en el que se analizan tendencias (Colmenares, 2021). En cuanto a las funciones u obligaciones que debe cumplir un observatorio, según Villegas (2016), se dice que este debe visualizar, promocionar, sistematizar, articular, difundir y fortalecer la economía social y solidaria, analizar, generar y evaluar la información sobre las políticas públicas, dar visibilidad y apoyar la producción de conocimiento, consolidar puntos de encuentro e intercambio de experiencias y desarrollar actividad económica y captar datos.

En cuanto a los recursos y los servicios, lo conforman: un marco jurídico, consejo directivo, legislación, políticas públicas, organismos de integración, área de prensa y documentos y enlaces. Por otro lado, debe ofrecer servicios de formación, incubación (construcción de circuitos económicos, cooperación social), consulta de políticas públicas (organismos, programas y destinatarios), estudios e investigaciones (Norchales y Abreu, 2016).

Economía solidaria: su definición integra las entidades de economía solidaria (cooperativas, mutualidades y asociaciones), que tienen una naturaleza legal no capitalista, puesto que son organizaciones democráticas que no condicionan sus decisiones al capital aportado de sus miembros (Pérez y Etxezarreta, 2015). La economía solidaria se ha constituido como una alternativa de producción por situaciones particulares de las necesidades de las comunidades, la cual busca un equilibrio entre el bien común y la libertad personal (Velez-Tamayo, 2014). Entre los problemas que enfrenta la sociedad colombiana, se encuentra el bajo nivel de solidaridad, la falta de asociatividad y el individualismo, debilitando la potencialidad de convivir y producir colectivamente, por lo que resulta indispensable crear políticas públicas y ejecutar estrategias que fomenten la cultura corporativa y la solidaridad en Colombia, con el fin de desarrollar la economía y la sociedad, impactando especialmente en la reducción de la pobreza, la creación de empleo y la integración, ya que las organizaciones de la economía solidaria son consideradas como una ayuda para construir un mundo mejor (Serna-Gómez y Rodríguez-Barrero, 2016).

Portal web: según Villacís (2017), la palabra portal como tal significa "puerta grande"; dicho significado es preciso para hacer referencia a su función y finalidad, ya que se define el portal web como un sitio web 
mediante el cual se puede acceder a una multitud de recursos o servicios, entre los que se destacan documentos, blogs, buscadores, foros, programas, compra y venta electrónica, entre otros.

Diseño web: según Aubry (2014), el concepto nace a principio de los años noventa; ahora bien se suele confundir diseño del desarrollo web, son partes que tienen lugar al elaborar un sitio web, pero son partes distintas del proyecto, aunque no se encuentren completamente separadas, el diseño web se encarga de la experiencia de usuario, da relevancia a la parte gráfica, como también a determinar los objetivos del proyecto, las necesidades del usuario, la arquitectura web y el bloque de contenidos; es la encargada de estudiar las funciones y la navegabilidad, con el objetivo de elaborar wireframes o prototipos, por lo que, en general, es la fase que se encarga de la navegación, usabilidad, interacción, arquitectura de la información y la parte gráfica de la web (Barba, 2014).

Se debe tener en cuenta que cuando el contenido y las funciones son complejas o cuando el tamaño de la webapp incluye cientos o miles de objetos de contenido, funciones y clases de análisis y cuando el éxito de la webapp tenga influencia directa en el éxito del negocio, el diseño no puede ni debe tomarse a la ligera, por lo tanto, una vez concluido el análisis de la interfaz con todas las tareas (objetos y acciones) requeridas por el usuario final, iniciará la actividad de diseño de la interfaz, un proceso iterativo, en el que se elabora y se refina la información desarrollada. Por esto, se sugieren que se desarrollen las siguientes etapas: definir objetos y acciones de la interfaz (operaciones), definir eventos (acciones del usuario), modelar este comportamiento, ilustrar cada estado de la interfaz como lo vería en la realidad el usuario final e indicar cómo interpreta el usuario el estado del sistema a partir de la información provista a través de la interfaz (Pressman, 2010).

Requerimientos funcionales: según Mariño et al. (2012), los requerimientos funcionales describen lo que el sistema debe hacer y dependen del tipo de software por desarrollar. Son declaraciones de los servicios que proveerá el sistema, de manera en que este reaccionará en situaciones particulares.

Requerimientos no funcionales: de acuerdo con Mariño et al. (2012), son limitaciones de los servicios o funciones ofrecidos por el sistema. Incluyen restricciones de tiempo, sobre el proceso de desarrollo, estándares, etc., no se refieren directamente a las funciones específicas que entrega el sistema, sino las propiedades emergentes de este como la fiabilidad, la respuesta en el tiempo y la capacidad de almacenamiento.

Capas de la arquitectura de software: según Pressman (2010), describe una infraestructura que permite que una aplicación basada en el web alcance sus objetivos, por lo que se sugiere una arquitectura de diseño en tres capas que desmiembra la interfaz de navegación y el comportamiento de la aplicación. El modelo vista controlador es uno de los modelos sugeridos para la infraestructura de las webapps que desacopla la interfaz de usuario de sus funciones y contenido.

El modelo, también denominado "objeto de modelo", contiene todos los objetos de contenido y todas las funciones de procesamiento que son específicas de la aplicación, por otro lado, la vista contiene todas las funciones específicas de la interfaz y permite la presentación de contenido y
AVANCES DE INVESTIGACIÓN

DISEÑO DE UN PORTAL WEB QUE PERMITA ACCEDER A LOS RECURSOS Y SERVICIOS DEL OBSERVATORIO DE ECONOMÍA SOLIDARIA 
AVANCES DE INVESTIGACIÓN

DISEÑO DE UN PORTAL

WEB QUE PERMITA

ACCEDER A LOS

RECURSOS Y SERVICIOS

DEL OBSERVATORIO DE

ECONOMÍA SOLIDARIA lógica de procesamiento y todas las funciones de procesamiento que requiere el usuario final. Por último, está el controlador que dirige el acceso al modelo y la vista, y coordina el flujo de datos entre ellos.

Herramientas: según Pressman (2010), las herramientas ágiles favorecen el uso de herramientas que permiten la creciente rápida del entendimiento. Algunas de las herramientas son sociales y comienzan en la etapa de reclutamiento, otras son tecnológicas y ayudan a que los equipos distribuidos simulen una presencia física. Las herramientas físicas permiten que las personas las manipulen, por lo que son clave para la contratación del personal adecuado, la colaboración del equipo, la comunicación con los participantes y la administración indirecta.

\section{RESULTADOS}

\section{ASPECTOS TECNOLÓGICOS}

Se pudieron establecer los aspectos tecnológicos requeridos para el diseño de un portal web que permita acceder a los recursos y servicios del observatorio de economía solidaria, garantizando la conservación y la actualización permanente de la información, a partir de la revisión bibliográfica de artículos científicos con búsquedas a partir de las palabras clave, como: observatorio virtual, observatorio digital, observación web, portales de conocimiento, observatorio social, observatorio tecnológico, en diversas bases de datos de acceso abierto (Scopus, Mendeley y Google académico) en las que se declaró un tiempo de 10 años de antigüedad.

Los aspectos tecnológicos definidos para el diseño del portal web del observatorio de economía solidaria se dividieron en requerimientos funcionales (divulgación de información, procesos y participantes), no funcionales y otros aspectos (etapas, arquitectura, herramientas y aspectos por medir) como se puede observar en la figura 1.

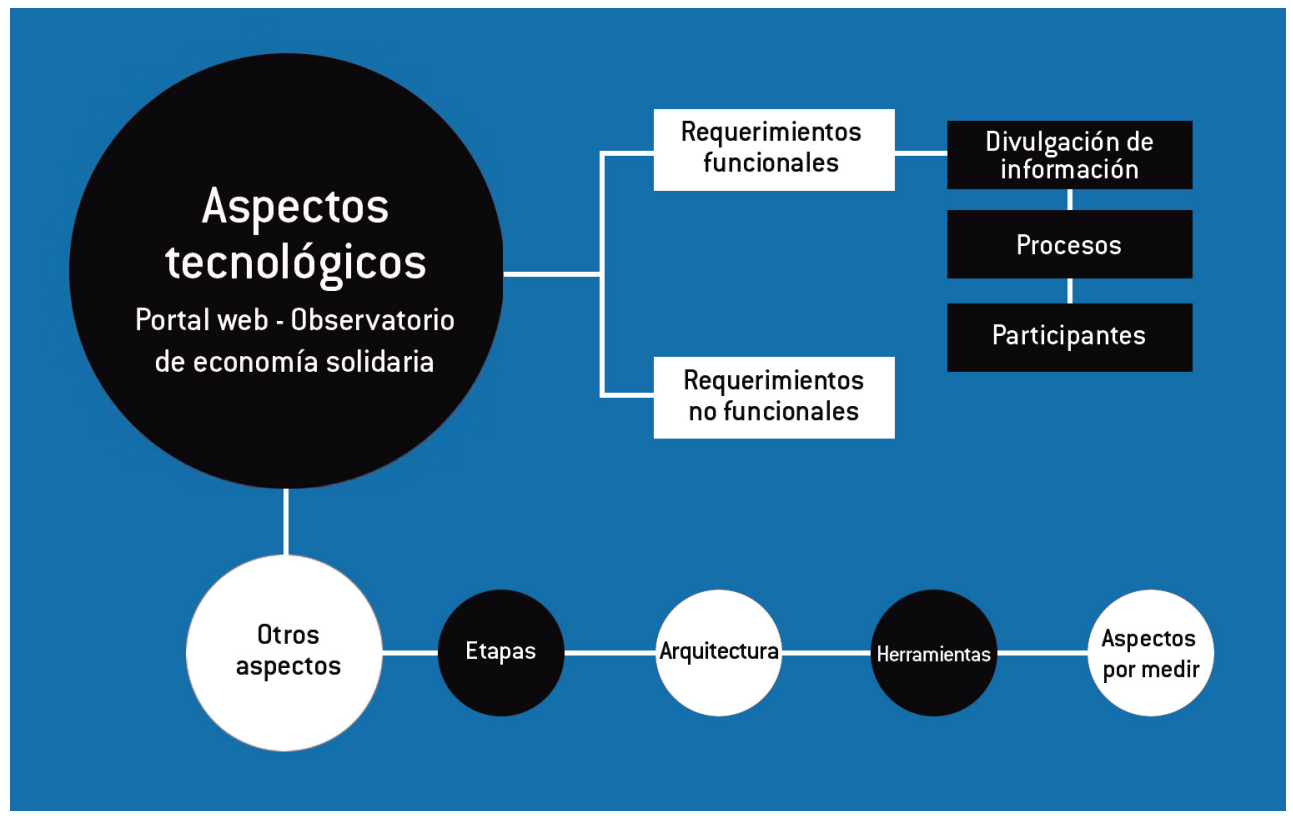

FIGURA 1. Aspectos tecnológicos. Elaboración propia. 
Dentro de los requerimientos funcionales, se pudo determinar que es importante divulgar: estadísticas e indicadores, normativas o políticas públicas, apoyo a empresarios, bases de datos e investigaciones, en cuanto a los procesos que se deben realizar, es necesario: monitorear, difundir, fomentar y observar, realizar comparaciones en el tiempo a través de un análisis cuantitativo como se puede apreciar en la figura 2 requerimientos funcionales.

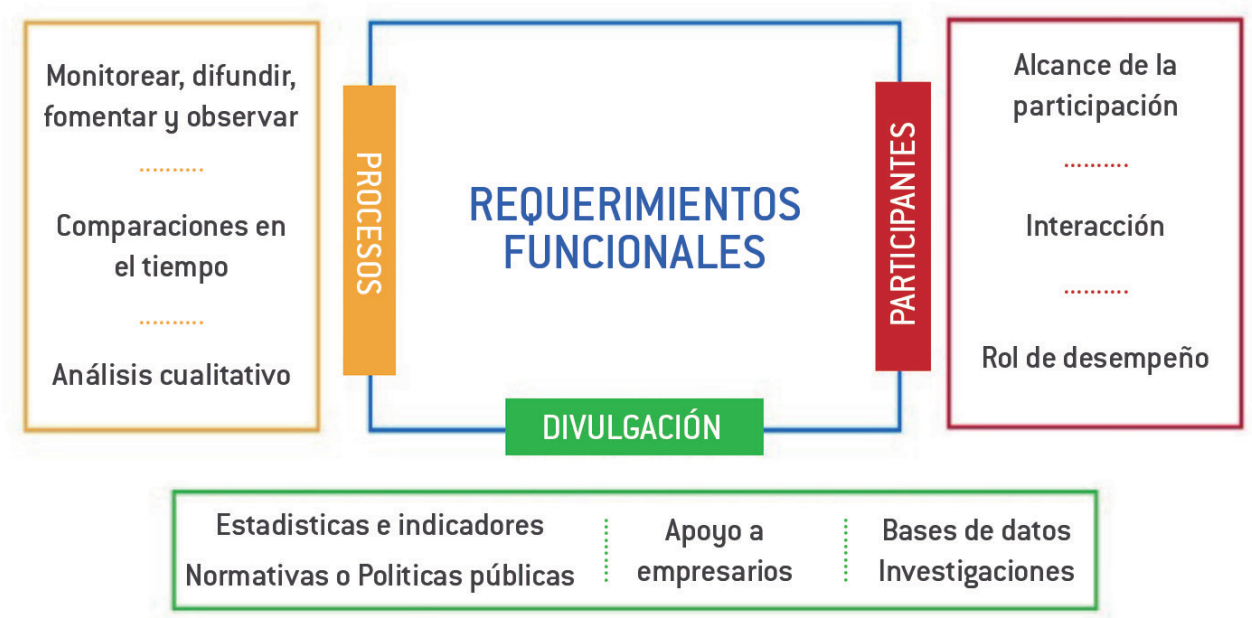

DISEÑO DE UN PORTAL WEB QUE PERMITA ACCEDER A LOS RECURSOS Y SERVICIOS DEL OBSERVATORIO DE ECONOMÍA SOLIDARIA

FIGURA 2. Requerimientos funcionales. Elaboración propia.

Con respecto a los requerimientos no funcionales, se pudo conocer que el diseño del portal se debe tener en cuenta, entre otros aspectos, que este soporte de consultas de acceso remoto, una rápida navegación y despliegue de información, compatibilidad con distintos navegadores y tener un procesamiento eficiente, como se puede apreciar en la figura 3.

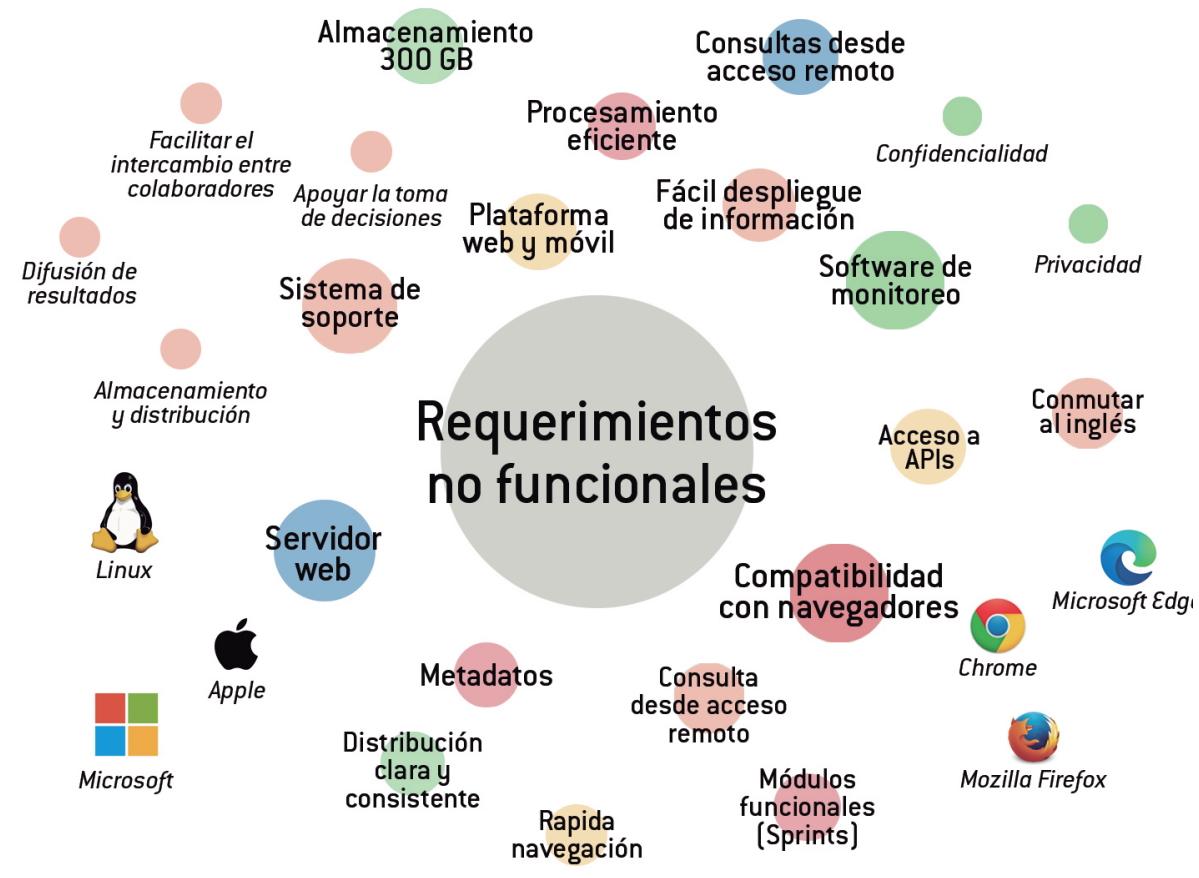

FIGURA 3. Requerimientos no funcionales. Elaboración propia. 
AVANCES DE INVESTIGACIÓN

DISEÑO DE UN PORTAL

WEB QUE PERMITA

ACCEDER A LOS

RECURSOS Y SERVICIOS

DEL OBSERVATORIO DE

ECONOMÍA SOLIDARIA
Por último, pero no menos importante, son otros aspectos tecnológicos que se deben considerar al momento de desarrollar el portal web para el observatorio de economía solidaria, como es definir las capas de la arquitectura, las herramientas por utilizar, el personal y los aspectos por medir (niveles de pobreza, exclusión social, derechos humanos, diversidad cultural, empleo, educación entre otros; ver figura 4).

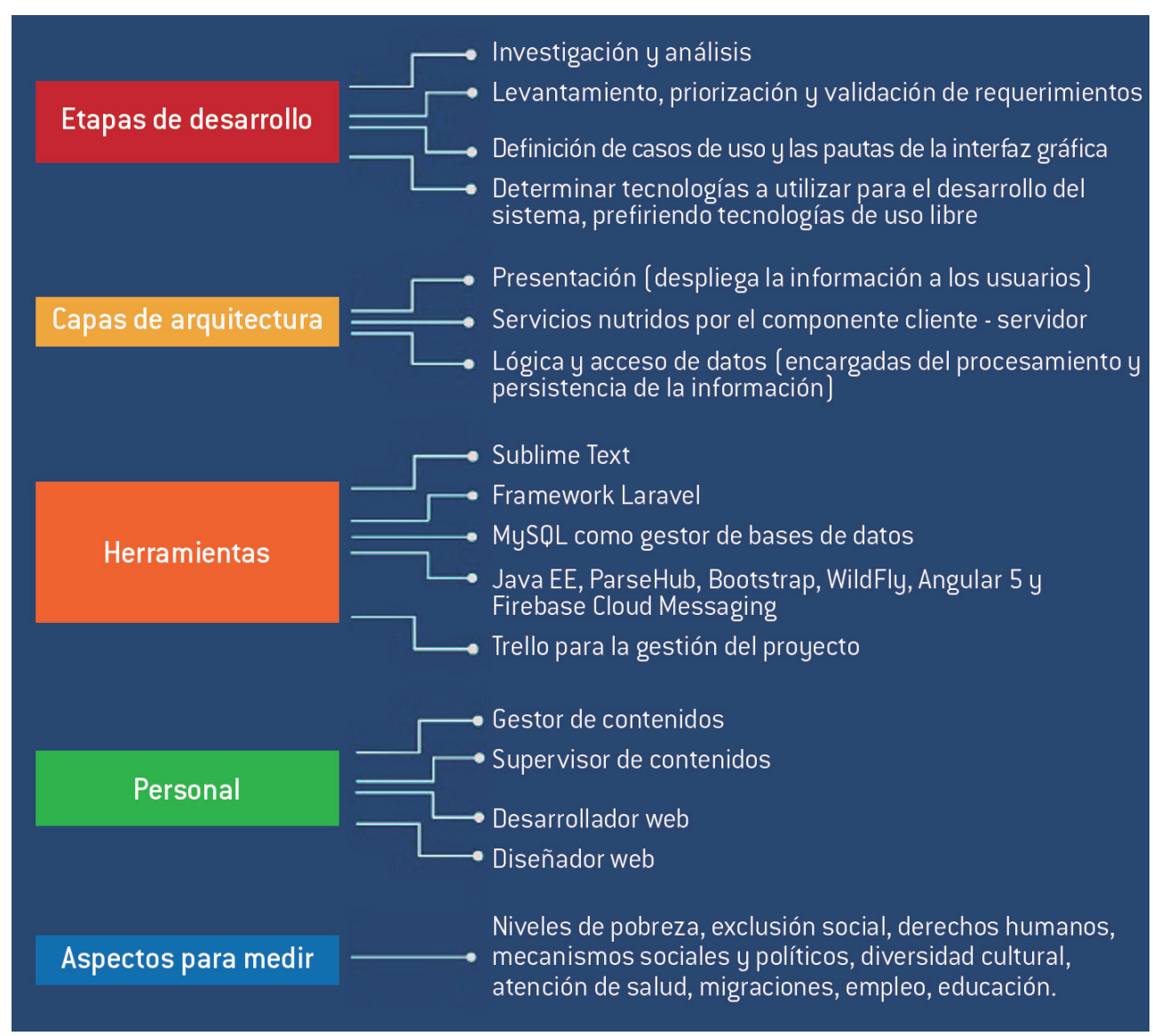

FIGURA 4. Otros aspectos tecnológicos. Elaboración propia.

Además de los aspectos tecnológicos descritos, se pudo conocer sobre los beneficios para los participantes:

- Conocer su propia identidad para satisfacer sus necesidades.

- Elevar el nivel de vida de las comunidades.

- Mejorar la gobernabilidad y la gobernanza.

- Comprender la aplicación de su filosofía.

- Demostrar con hechos a través de la investigación la importancia del sector.

- Innovación social: innovación tecnológica para beneficios sociales.

- Reducir los costos de infraestructura big data.

- Involucrar empresas más pequeñas a la innovación impulsada por los datos.

- Alfabetización digital. 
- Empoderar al público, en general, para acceder a fuentes de datos sobre asuntos de ciencia, gobierno y economía.

Como también existen desafíos de implementar un observatorio:

- Rendimiento de los algoritmos.

- Aprendizaje automático.

- Amigabilidad de la interfaz de usuario.

- Recolección de los datos.

- Actualización automática de la información.

\section{CARACTERIZACIÓN DE OBSERVATORIOS DE ECONOMÍA SOLIDARIA}

Se procedió a caracterizar cinco observatorios de economía solidaria internacionales, teniendo en cuenta los aspectos tecnológicos identificados con el fin de detectar sus mejores aspectos y tomarlos como referencia para el prototipo no operacional o modelo no funcional del observatorio de economía solidaria, con el cual se pudieran probar aspectos del diseño. Los observatorios caracterizados fueron los siguientes: observatorio del sur de la economía social y solidaria, observatorio de políticas públicas de economía popular, social y solidaria, observatorio nacional de economía solidaria y cooperativismo, observatorio iberoamericano del empleo y la economía social y cooperativa, observatorio español de la economía social.

La caracterización de los observatorios permitió conocer sobre las posibles organizaciones aliadas que debe considerar el observatorio, como se puede ver en la figura 5 .

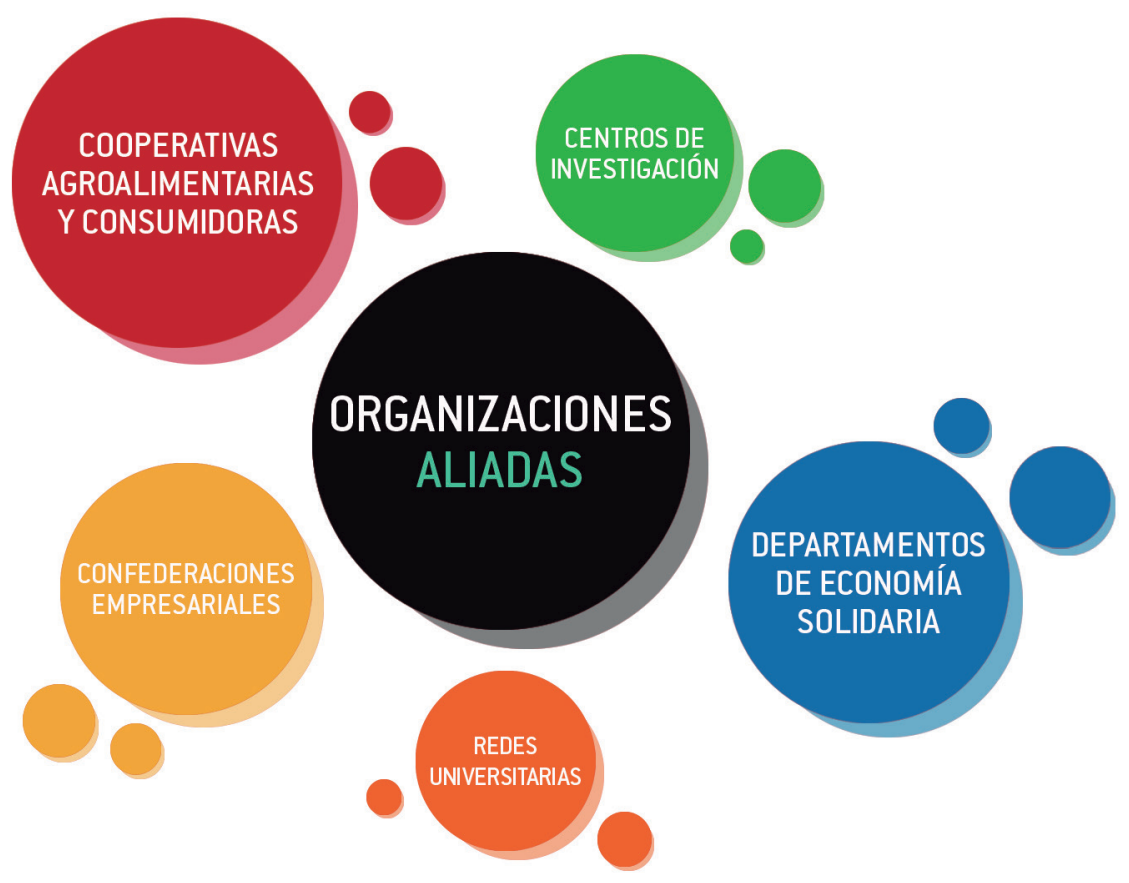


Se definieron también las funciones u obligaciones del observatorio, entre ellas: conformar espacios de referencia, dar visibilidad y apoyo a la producción de conocimiento, captar datos, analizar, generar y evaluar WEB QUE PERMITA información sobre políticas públicas (ver figura 6).

ACCEDER A LOS

RECURSOS Y SERVICIOS

DEL OBSERVATORIO DE ECONOMÍA SOLIDARIA

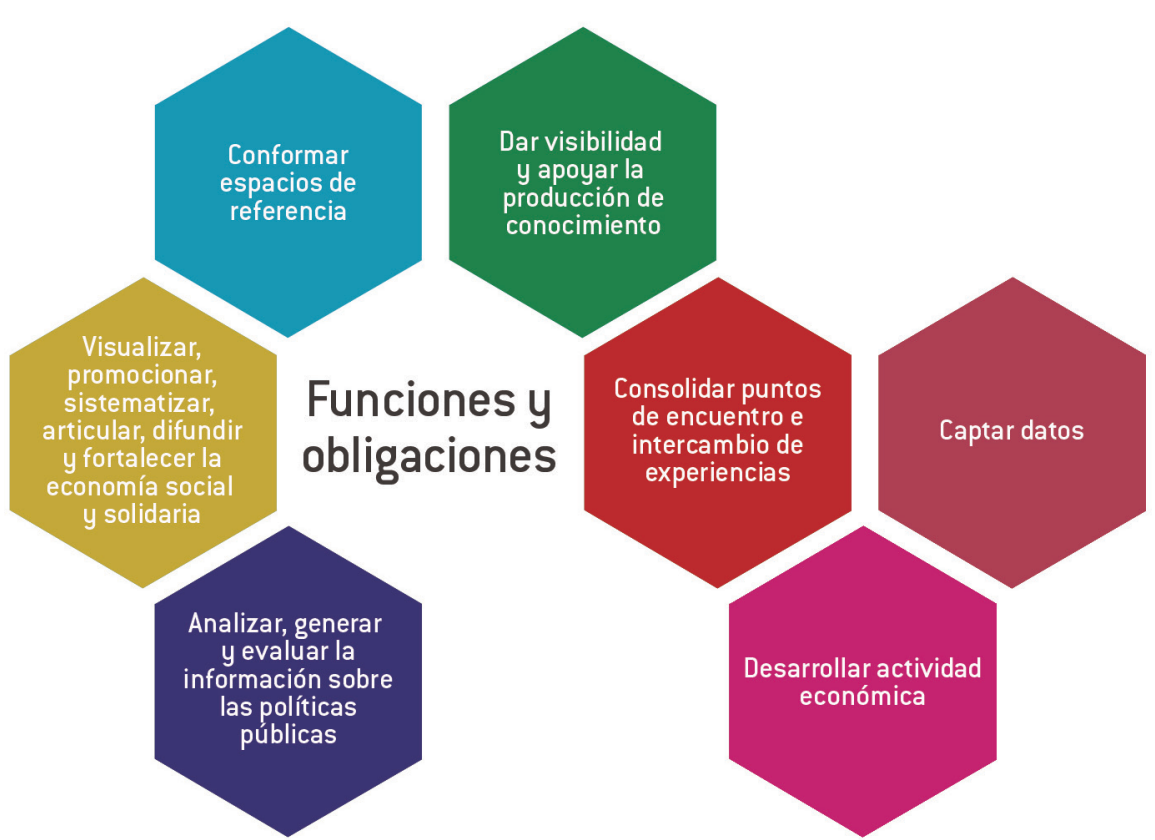

FIGURA 6. Funciones u obligaciones. Elaboración propia.

Finalmente, a partir de la caracterización de los observatorios se pudo establecer los posibles recursos y servicios que se pudiesen integrar al portal web del observatorio de economía solidaria, entre ellos la formación, la incubación social, mercado territorial, estudios e investigaciones, mapa interactivo de organizaciones aliadas y divulgación de información de economía solidaria, como se presenta en la tabla 1.

TABLA 1. Recursos o servicios

\section{RECURSOS / SERVICIOS}

Formación: cursos y talleres. Incubación social (construcción de circuitos económicos, cooperación social).

Mercado territorial.

Estudios e investigaciones.

Fortalecimiento de desarrollo del campo Ess. Cooperativas y sociedades laborales. Mapa interactivo de organizaciones aliadas.
Divulgar información economía solidaria:

Políticas públicas.

Organismos de integración.

Documentos, enlaces y boletines.

Marco jurídico.

Legislación.

Informes.

Buenas prácticas.

Datos y estadísticas.

Perfiles de territorio.

NOTA: elaboración propia.

PROTOTIPO NO OPERACIONAL O MODELO NO FUNCIONAL DEL PORTAL WEB DEL OBSERVATORIO DE ECONOMIA SOLIDARIA

A partir de procesos de diseño y validación de usabilidad en el diseño de interfaces, la cual consistió en la aplicación de un formulario con 
las siguientes preguntas por parte de cuatro investigadores del proyecto observatorio de impacto social, económico y ambiental de la economía solidaria en Colombia. El 100\% estuvo de acuerdo en que el diseño general de la página tiene lectura y navegación fácil y se accede al contenido con facilidad; el $75 \%$ estuvo de acuerdo en que la interfaz fue sencilla de aprender y le ayuda a ser más productivo su trabajo; por otro lado, solo el $50 \%$ estuvo de acuerdo en que la interfaz dispone de todas las potencialidades que necesita y el $25 \%$ estuvo de acuerdo en que interactuar con la interfaz fue una experiencia frustrante.

Además de la validación de usabilidad, se propuso calificar la prioridad de los contenidos de los distintos menús del diseño de la interfaz, entre ellos, nuestra entidad, divulgación, recursos, servicios, indicadores y aliados, obteniendo en promedio una prioridad de cuatro para todos. Finalmente, se obtuvo aprobación por parte del equipo investigador la aprobación para el prototipo no operacional del portal web del observatorio de economía solidaria, que consiste en dos barras de menús principales, la primera ubicada en la parte superior con los apartados: quiénes somos, qué hacemos, cómo lo hacemos y el inicio de sesión. El segundo menú ubicado a la izquierda de la pantalla con los apartados: indicadores, servicios y recursos, como se puede visualizar en la figura 8.

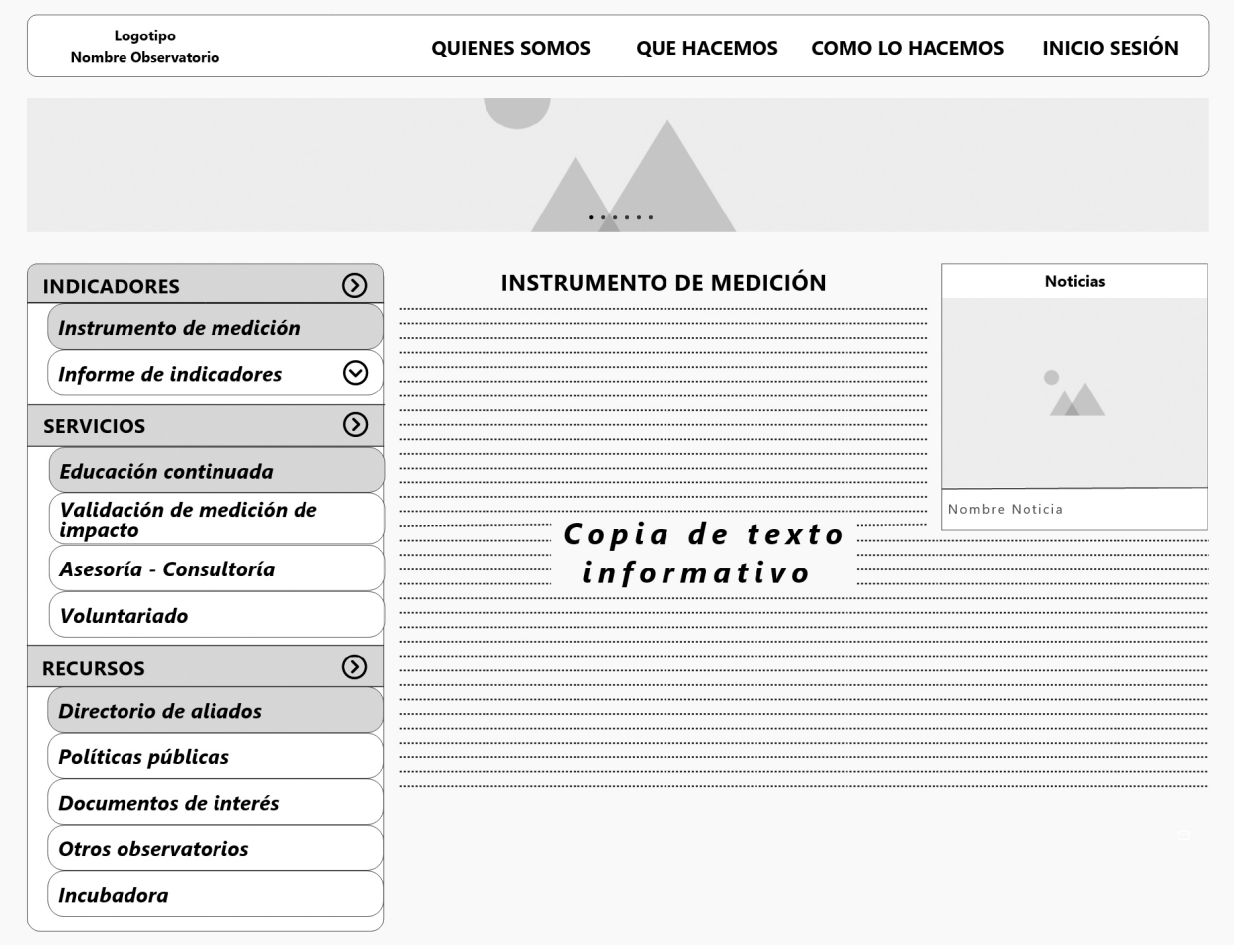
Direccion: $x \times x x x x$
Teléfono: $x x x x x x$
Correo Institucional: $x x x x x x$
0000 
La presente investigación después de realizar un proceso de exploración y análisis de las cualidades de los observatorios permitió establecer los aspectos tecnológicos necesarios para el diseño de un portal web que permita acceder a los recursos y servicios del observatorio de economía solidaria, entre ellos, los requerimientos funcionales y no funcionales, destacando que los requerimientos funcionales que se deben implementar para el observatorio son aquellos que ayuden al cumplimiento de sus funciones u obligaciones como lo son:

- La divulgación de información de economía solidaria: estadísticas e indicadores, normatividad o políticas públicas, organismos de integración, buenas prácticas, perfiles de territorio, noticias sobre la gestión socioeconómica y ambiental, nuevas investigaciones y el apoyo a empresarios.

- La realización de procesos: visualizar, monitorear, sistematizar, articular, difundir, fomentar, observar, fortalecer las diferentes organizaciones que ejercen actividades para el beneficio colectivo o social.

- Realizar comparaciones en el tiempo, consolidar puntos de encuentro e intercambio de experiencias y analizar cualitativamente relatos y revisiones documentales, como también promocionar sus servicios (formación, incubación social, mercado territorial, investigación, mapa interactivo de organizaciones aliadas).

Para la implementación del portal web, se deben tener en cuenta los requerimientos no funcionales, entre los cuales se destacan: contar con una plataforma de colaboración electrónica basada en la web y móvil compatible con diferentes navegadores, puntos de acceso a la interfaz de programación de aplicaciones (API), metadatos, admitir consultas distribuidas desde acceso remoto, contar con un software que monitoree constantemente la privacidad y la confidencialidad del observatorio, tener registro en URL, obtener un almacenamiento mínimo de 100 gigabytes para los primeros dos años para programas y aplicaciones y un almacenamiento de 200 gigabytes para publicación de materiales y metadatos, tener un servidor web que permita su ejecución desde todas las plataformas (Windows, Linux y Mac), desarrollar un sistema de información que dé soporte al almacenamiento de la información, la difusión de resultados, el procesamiento eficiente y apoyo a la toma de decisiones.

Entre otros aspectos tecnológicos identificados es importante que se planifiquen las fases para su desarrollo, además del presente proceso de investigación y análisis (investigación de observatorios para determinar principales funcionalidades), el levantamiento, priorización y validación de requerimientos, la definición de casos de uso y las pautas de la interfaz gráfica y determinar las tecnologías que se van a utilizar para el desarrollo del sistema, prefiriendo tecnologías de uso libre. Es fundamental definir las capas de la arquitectura de software (presentación, servicios, lógica y acceso de datos), como también el personal necesario para el desarrollo y su posterior mantenimiento (gestor de contenidos, supervisor de 
contenidos, desarrollador web y diseñador web). Adicional a lo anterior, no se pueden desconocer los desafíos a los que se enfrenta el desarrollo de un portal web al servicio del observatorio de economía solidaria: el rendimiento de los algoritmos, el aprendizaje automático, la amigabilidad de la interfaz de usuario, la recolección de los datos y la actualización automática de la información.

\section{REFERENCIAS}

Altamirano, E. J., Benavidez, W. J., Martínez, R. S. y Rodríguez, M. R. (2018, junio). Aplicación web para un observatorio socioeconómico que permite monitorear indicadores desde diferentes instituciones. Obtenido de Aplicación web para un observatorio socioeconómico que permite monitorear indicadores desde diferentes instituciones. https:// bbibliograficas.ucc.edu.co:4046/catalogue/7f5f40bb-1653-3ca8-8c5 a-37193b607204/

Aubry, C. (2014). HTML5y Css3:parasitios condiseñowebresponsive. Ediciones ENI. https://books.google.es/books?hl=es\&lr=\&id=ys5PaRPiEwwC\&oi=fnd\&pg $=$ PA $9 \& d q=$ dise $\% C 3 \% B 10+$ web\&ots=oAEWVlbr8J\&sig=j0_ hHOFdQ5QKx2g1PCk7mZCgyY\#v=onepage\&q=dise $\%$ C3\%B1o\%20 web\&f=false

Barba, J. (2014). Diseño y desarrollo web (Tesis de grado). Univesitat Politécnica de Valencia, Valencia, España. https://riunet.upv.es/bitstream/ handle/10251/49757/MEMORIA_Barba\%2520Soler\%252C\%2520Juan\%2520Pedro.pdf?sequence=1

Briones-Veliz, T., Carvajal-Avila, D. y Sumba-Bustamante, R. (2021). Utilidad del benchmarking como estrategia de mejora empresarial. Polo del Conocimiento: Revista científico-profesional, 6(3), 2026-2044. https://dialnet.unirioja.es/servlet/articulo?codigo $=7926897$

Cardozo, V., Sosa, S., Vilaboa, C., Sosa, R. y Rienzi, B. (2018, julio). Observatorio de tecnologías de la información geográfica (Tesis de grado). Universidad de la República, Montevideo, Uruguay. https://www.colibri.udelar.edu.uy/jspui/bitstream/20.500.12008/20023/1/2822.pdf

Colmenares, W. (2021). Aproximación al concepto de observatorio editorial: recomendaciones para su creación. Revista General de Información y Documentación, 31(1), 119-147. https://dx.doi.org/10.5209/ rgid.73471

Esteban-Regino, E. M. (2016). Importancia del desarrollo de habilidades académicas como estrategia para el inicio de la formulación y evaluación de proyectos tecnológicos (Documento de docencia N. ${ }^{\circ} 17$ ). Ediciones Universidad Cooperativa de Colombia. Doi: https://repository.ucc. edu.co/handle/20.500.12494/16567

Hernández, R., Fernández, C. y Baptista, P. (2006). Metodología de la investigación. Mc Graw Hill.

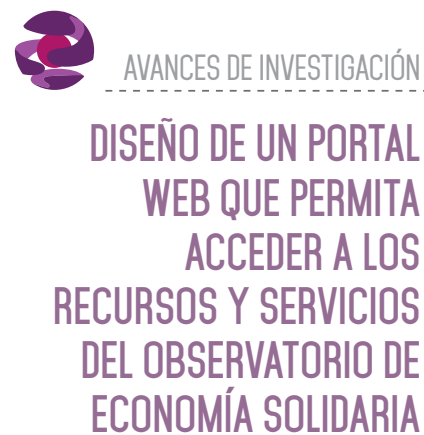


AVANCES DE INVESTIGACIÓN

DISEÑO DE UN PORTAL

WEB QUE PERMITA

ACCEDER A LOS

RECURSOS Y SERVICIOS

DEL OBSERVATORIO DE

ECONOMÍA SOLIDARIA
Kendall, K. E. y Kendall, J. E. (2011). Análisis y diseño de sistemas (8 ${ }^{a}$ ed.). Pearson Educación.

Mariño, S., Godoy, M., Acevedo, P., Gómez, J. y Vázquez, L. F. (2012, diciembre). Accesibilidad en la definición de requerimientos no funcionales. Accesibilidad en la definición de requerimientos no funcionales. https://www.redalyc.org/pdf/904/90426810009.pdf

Norchales, Y. M. y Abreu, M. B. (2016, abril). Caracterización de los observatorios como plataformas para la gestión de la vigilancia tecnológica en el sector de la Educación Superior. Enl@ce: revista Venezolana de Información, Tecnología y Conocimiento, 13(1), 11-27. https:// www.mendeley.com/catalogue/605f10c2-4a99-34ac-987b-93dcc9274e8b/?utm_source=desktop\&utm_medium =1.19.8\&utm_ campaign=open_catalog \&userDocument Id $=\% 7$ Bd 3dd9786-40be-4cc6-8f52-3fa68ccb7bef\%7D

Observatorio de Economía social y solidaria. (s. f.). Obtenido de Observatorio de Economía Social y Solidaria: http://observatorioess.org.ar/

Observatorio de políticas públicas de economía popular, social y solidaria. (s. f.). http://oppepss.ungs.edu.ar/

Observatorio español de la economía Solidaria. (s. f.). http://www.observatorioeconomiasocial.es/

Observatorio Iberoamericano del empleo de la economía social y cooperativa. (s. f.). http://www.oibescoop.org/

Observatorio nacional de economía solidaria y cooperativismo. (s. f.). https://ecosol.dieese.org.br/

Pérez, J. y Etxezarreta, E. (2015). Sobre el concepto de economía social y solidaria: aproximaciones desde Europa y América Latina. Revista de Economía Mundial, 40, 123-144 https://dialnet.unirioja.es/servlet/ articulo?codigo $=5199917$

Pérez, Y. I. (2016). Diseño de un observatorio de gestión ambiental: una alternativa de apoyo al desarrollo local (Tesis de maestría). Instituto Superior Minero Metalúrgico Dr. Antonio Núñez Jiménez. http://ninive. ismm.edu.cu/bitstream/handle/123456789/3492/YoneidisHernándezPérez.pdf?sequence=1\&isAllowed=y

Pressman, R. (2010). Ingeniería de software un enfoque práctico. Mc Graw Hill. http://cotana.informatica.edu.bo/downloads/ld-Ingenieria.de. software.enfoque.practico.7ed.Pressman.PDF

Rivera, A. y Aranzales, E. R. (2016, mayo). El observatorio: una herramienta para el sector social, cooperativo y solidario en la región del Tolima, Colombia. Cooperativismo \& Desarrollo, 109. https://doi. org/10.16925/co.v24i109.1510

Sepúlveda, A. J. y Fernández, G. R. (2017). Planteamiento para el diseño e implementación de un observatorio enfocado a la economía solidaria en la 
ciudad de Cali (Tesis de grado). Universidad ICESI, Cali, Colombia. https:// repository.icesi.edu.co/biblioteca_digital/bitstream/10906/83716/1/ TG01930.pdf

Serna-Gómez, H. y Rodríguez-Barrero, M. S. (2016). El sector solidario como alternativa para el desarrollo social e inclusivo en el posconflicto colombiano. Cooperativismo \& Desarrollo, 23(107). https://doi. org/10.16925/co.v23i107.1250

Soler, G. E. (2015, febrero). Una aproximación al concepto de observatorio social. Cultura Educación y Sociedad, 6(1). 93-108. https://revistascientificas.cuc.edu.co/culturaeducacionysociedad/article/view/758/ pdf_93

Tiropanis, T., Hall, W., Hendler, J. y Larrinaga, C. D. (2014). The Web Observatory: A Middle Layer for Broad Data. Obtenido de The Web Observatory: A Middle Layer for Broad Data. Big Data, 2(3),129133- https://www.liebertpub.com/doi/10.1089/big.2014.0035

Vélez-Tamayo, J. (2014). Construcción del concepto de economía solidaria: una mirada a la teoría económica comprensiva. Cooperativismo \& Desarrollo, 105, 17-29. https://doi.org/10.16925/co.v22i105.690.

Villacís, D. G. (2017). Desarrollo de un portal Web (Tesis de grado). Pontificia Universidad Católica de Ecuador, Quito, Ecuador. https://repositorio.pucesa.edu.ec/bitstream/123456789/1875/1/76378.pdf

Villegas, J. C. (2016, junio). Observatorio del hábitat de Bogotá: variables de referencia para su diseño. UVserva, 2, 41-48. https://uvserva. uv.mx/index.php/Uvserva/article/view/2375/4216

Voutssás, J. (2012). Observatorios web y portales del conocimiento. Investigación bibliotecnologica, 26(57). http://dx.doi.org/10.22201/ iibi.0187358xp.2012.57.33842

Wehn, U. y Evers, J. (2015). The Social Innovetion of ICT-Enabled Citizen Observatories to Increase E-Participation in Local Flood Risk Management. Technology in Society, 42, 187-198. https://doi.org/10.1016/j. techsoc.2015.05.002
DISEÑO DE UN PORTAL WEB QUE PERMITA ACCEDER A LOS RECURSOS Y SERVICIOS DEL OBSERVATORIO DE ECONOMÍA SOLIDARIA 

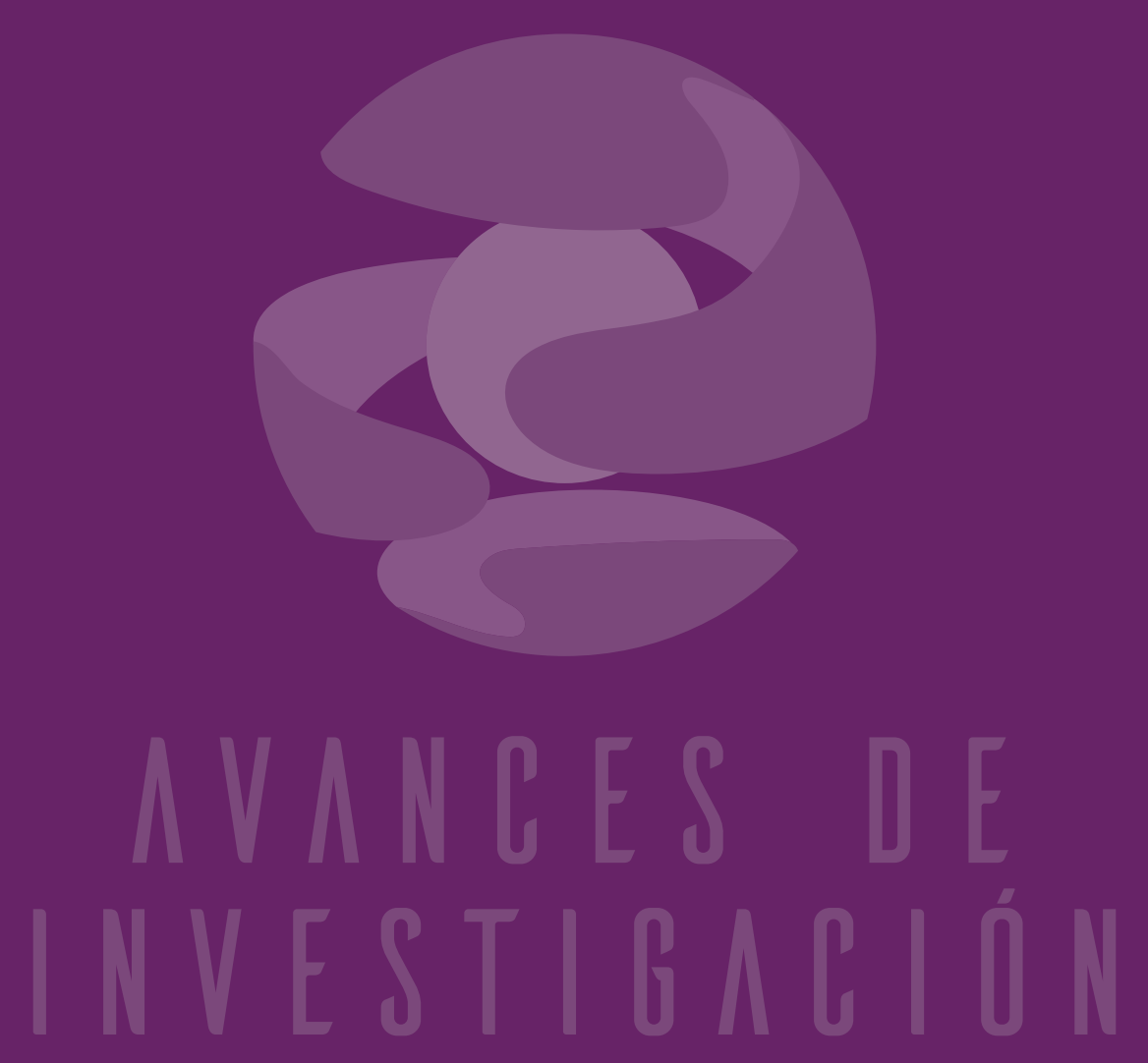